\title{
Transcribed-ultra conserved region expression is associated with outcome in high-risk neuroblastoma Paola Scaruffi ${ }^{*}$, Sara Stigliani ${ }^{1}$, Stefano Moretti ${ }^{2}$, Simona Coco ${ }^{1}$, Carla De Vecchi $^{1}$, Francesca Valdora ${ }^{3}$, Alberto Garaventa ${ }^{4}$, Stefano Bonassi ${ }^{5}$ and Gian Paolo Tonini ${ }^{1}$
}

\author{
Address: ${ }^{1}$ Translational Paediatric Oncology, National Cancer Research Institute (IST), Largo R. Benzi 10, Genoa, 16132, Italy, ${ }^{2}$ Molecular \\ Epidemiology, National Cancer Research Institute (IST), Largo R. Benzi 10, Genoa, 16132, Italy, ${ }^{3}$ Department of Oncology and Genetics (DOBIG), \\ University of Genoa, Largo R. Benzi 10, Genoa, 16132, Italy, ${ }^{4}$ Department of Hematology-Oncology, Gaslini Institute, Largo G. Gaslini 5, Genoa, \\ 16148, Italy and ${ }^{5}$ Clinical and Molecular Epidemiology, IRCCS San Raffaele Pisana, Via della Pisana 235, Roma, 00163, Italy \\ Email: Paola Scaruffi* - paola.scaruffi@istge.it; Sara Stigliani - sara.stigliani@istge.it; Stefano Moretti - stefano.moretti@istge.it; \\ Simona Coco - simona.coco@istge.it; Carla De Vecchi - carla.devecchi@istge.it; Francesca Valdora - francesca.valdora@istge.it; \\ Alberto Garaventa - albertogaraventa@ospedale-gaslini.ge.it; Stefano Bonassi - stefano.bonassi@sanraffaele.it; \\ Gian Paolo Tonini - gianpaolo.tonini@istge.it \\ * Corresponding author
}

Published: I5 December 2009

BMC Cancer 2009, 9:44I doi:10.1 186/|47|-2407-9-44|

This article is available from: http://www.biomedcentral.com/|47/-2407/9/44 |

(c) 2009 Scaruffi et al; licensee BioMed Central Ltd.

This is an Open Access article distributed under the terms of the Creative Commons Attribution License (http://creativecommons.org/licenses/by/2.0), which permits unrestricted use, distribution, and reproduction in any medium, provided the original work is properly cited.

\begin{abstract}
Background: Neuroblastoma is the most common, pediatric, extra-cranial, malignant solid tumor. Despite multimodal therapeutic protocols, outcome for children with a high-risk clinical phenotype remains poor, with long-term survival still less than $40 \%$. Hereby, we evaluated the potential of non-coding RNA expression to predict outcome in high-risk, stage 4 neuroblastoma.

Methods: We analyzed expression of 48I Ultra Conserved Regions (UCRs) by reverse transcription-quantitative real-time PCR and of 723 microRNAs by microarrays in 34 high-risk, stage 4 neuroblastoma patients.

Results: First, the comparison of 8 short- versus 12 long-term survivors showed that 54 UCRs were significantly $(P<0.0491)$ over-expressed in the former group. For 48 Ultra Conserved Region (UCRs) the expression levels above the cut-off values defined by ROC curves were strongly associated with good-outcome (OS: $0.000 \mathrm{I}<P<$ 0.0185 , EFS: $0.0001<P<0.0491$ ). Then we tested the Transcribed-UCR (T-UCR) threshold risk-prediction model on an independent cohort of 14 patients. The expression profile of $28 \mathrm{~T}$-UCRs was significantly associated to prognosis and at least I 5 up-regulated T-UCRs are needed to discriminate $(P<0.000 \mathrm{I})$ short- from long-survivors at the highest sensitivity and specificity (94.12\%). We also identified a signature of 13 microRNAs differently expressed between long- and short-surviving patients. The comparative analysis of the two classes of non-coding RNAs disclosed that 9 T-UCRs display their expression level that are inversely correlated with expression of 5 complementary microRNAs of the signature, indicating a negative regulation of T-UCRs by direct interaction with microRNAs. Moreover, 4 microRNAs down-regulated in tumors of long-survivors target 3 genes implicated in neuronal differentiation, that are known to be over-expressed in low-risk tumors.
\end{abstract}

Conclusions: Our pilot study suggests that a deregulation of the microRNA/T-UCR network may play an important role in the pathogenesis of neuroblastoma. After further validation on a larger independent set of samples, such findings may be applied as the first T-UCR prognostic signature for high-risk neuroblastoma patients. 


\section{Background}

Neuroblastoma (NB) is a pediatric tumor of the sympathetic nervous system characterized by a remarkable heterogeneous clinical behavior [1]. Patients with localized NB have a favorable outcome, and in infants with disseminated stage 4 tumor the progression of disease is generally halted by good response to therapy. Conversely, only $20-30 \%$ of children older than 12 months of age - or 18 months, according to a more recent classification [2] with a stage 4 tumor show progression free- and overallsurvival longer than 60 months, despite multimodal therapeutic protocols [3]. In recent years several prognostic signatures derived from gene expression profiles, DNA abnormalities, and microRNAs [4-9] have been proposed as sensitive indicators of tumor progression in NB patients. Yet, the major challenges remain to validate each gene classifier on independent patient cohorts, and to identify additional tumor-specific prognostic markers for improved risk estimation at the time of diagnosis, especially in high-risk NB patients.

Recently, Calin et al. [10] have demonstrated that a newly discovered class of Ultra Conserved Regions (UCRs) [11] is significantly altered at both DNA and RNA levels in adult chronic lymphocytic leukemias, colorectal and hepatocellular carcinomas. Moreover, they found that tumor-associated Transcribed-UCRs (T-UCRs) in leukemias are regulated by microRNAs, leading to the hypothesis that both coding and noncoding RNAs may define signatures associated with diagnosis, prognosis, and response to treatment. So far, there are no reports about the expression of T-UCRs in NB and there is presently only one published study on microRNA deregulation and NB prognosis [9]. Here we profiled both T-UCRs and microRNAs in a cohort of 34 high-risk NBs, with the aim to investigate their putative role as sensitive markers of outcome prediction in children with stage $4 \mathrm{NB}$, a major challenge to clinicians and researchers. Our pilot study suggests for the first time that a signature based on T-UCR expression is associated with good outcome in non infant patients diagnosed with metastatic $\mathrm{NB}$, and lets us assume putative complex regulatory interplays between T-UCRs and microRNAs in neuroblastoma. The potential to use this information must be tested in a larger, independent set of samples.

\section{Methods}

\section{Tumor samples}

Tumor specimens were collected at the onset of disease from 34 patients who were diagnosed with a primary NB between 1990 and 2006, and referred to the Gaslini Children Hospital, Genoa, Italy. The study was approved by Ethics committee of the Gaslini Children Hospital, and informed consent was obtained by all children's legal guardians. All patients were classified as stage 4 according to the International Neuroblastoma Staging System [12], and they were older than 1 year of age at time of diagnosis (average age: 39.2 months). Regarding the clinical course, patients were categorized into two subgroups: "short-survivors" (dead of disease within 36 months from diagnosis. Deaths due to toxicity were censored) and "longsurvivors" (alive with an overall survival time > 36 months). Long- and short-surviving patients did not differ by year of diagnosis. Patient and tumor characteristics are summarized in additional file 1: Table S1.

The study was initially conducted on a cohort of 20 patients: 8 short-survivors (median follow-up time: 18 months, range 6-34 months; median event-free surviving time: 14 months, range 5-29 months) and 12 long-survivors (median follow-up time: 86 months, range 39-164 months; median event-free surviving time: 72 months, range 22-164 months). None but 2 long-surviving patients (\#1558 and \#2056) had relapse or progression of disease. Case \#1558 had a local relapse after 22 months from diagnosis and is in complete remission with a follow-up time of 112 months. Patient \#2056 had a progression of disease after 70 months from diagnosis and at the time of analysis is alive with stable disease (follow-up time: 76 months).

Then, the T-UCR threshold risk-prediction model obtained from this group of patients was tested in an independent cohort of 14 patients. Within this set, 9 patients were included in the short-survivor group (median follow-up time: 18 months, range 1-35 months; event-free surviving time: 13 months, range 1-22 months) and 5 in the long-survivor one (median follow-up time: 53 months, range 40-67 months). The latter group of patients had neither relapse nor progression of disease.

All tumor samples were classified as Schwannian stromapoor neuroblastoma according to the International Neuroblastoma Pathology Committee [13], with at least $80 \%$ of neuroblasts. Amplification of the MYCN oncogene was present in tumors of 5 short- and 2 long-surviving patients.

\section{Reverse transcription quantitative real-time PCR (RT- qPCR)}

Total RNA was extracted from frozen tumors by PerfectPure $^{\mathrm{TM}}$ RNA Tissue Kit (5Prime, Hamburg, Germany), including RNase-free DNase I treatment. Total and small RNAs were quantified and quality control was assessed by RNA $6000 \mathrm{Nano}^{\circledast}$ and Small RNA ${ }^{\circledast}$ assays, respectively, on the 2100 Bioanalyzer (Agilent Technologies, Santa Clara, CA). Only samples with RNA integrity number $\geq 6.0$ were included in the study. RNA samples have been validated as DNA-free by a no-reverse transcription assay for Eukaryotic $18 \mathrm{~S}$ rRNA gene (VIC-labeled TaqMan Gene 
Expression assay, Applied Biosystems, Foster City, CA). RNA was amplified and reverse transcribed by WT-Ovation $^{\mathrm{TM}}$ RNA Amplification System kit (NuGEN Technologies, San Carlos, CA), and UCR transcripts were quantified by Transcribed Ultra Conserved Regions real-time PCR assays (PrimerDesign Ltd, Hants, UK) using SYBR ${ }^{\circledR}$ green detection chemistry. Reactions were setup in 96-whitewell Twin.tec ${ }^{\circledR}$ real-time plates (Eppendorf, Hamburg, Germany) by EpMotion 5070 Liquid Handling Workstation (Eppendorf) and performed in duplicate on RealPlex ${ }^{4}$ system (Eppendorf). Specificity of qPCR reactions has been assessed by melting curve analysis. According to the Minimum Information for Publication of Quantitative Real-Time PCR Experiments (MIQE) $[14,15]$, raw qPCR data and a checklist including technical details are submitted as Supplemental data (see additional file 2: Raw Cq data; additional file 3: MIQE checklist). The relative amount of each T-UCR to $18 \mathrm{~S}$ rRNA was determined using the equation $2^{-d C q}$, where $\mathrm{dCq}=\left(\mathrm{Cq}_{\mathrm{T}}\right.$ UCR $\left.-\mathrm{Cq}_{18 \mathrm{~S} \text { rRNA}}\right)$, as previously described [10].

\section{Statistical analyses}

Student's $t$-test was used to compare mean T-UCR expression levels between long- and short-surviving individuals after logarithmic transformation of original measures. All significance tests were two tailed. To identify those values providing the best separation between long- and shortsurvivors, T-UCR expression values were dichotomized according to Receiver Operating Characteristic (ROC) curves [16]. The threshold values identified with this procedure were used in subsequent survival analyses. Overall survival (OS) was defined as the time elapsed from diagnosis to cancer-related death or to the date of the last follow-up if the patient survived. Patients who survived were censored at the last date they were reported to be alive. Event free survival (EFS) was calculated from diagnosis to the date of tumor progression or relapse or to the date of the last follow-up if no event occurred. Survival curves were computed according to the Kaplan-Meier method. The Cox proportional-hazards regression model was used to study in a multivariate setting the effect of MYCN status (amplified, not amplified) as potential confounder. A backward selection approach was used and only variables with $P<0.05$ were retained in the model. The MedCalc ${ }^{\circledR}$ software (Mariakerke, Belgium) was used for statistical analyses.

\section{Oligonucleotide array CGH}

Array CGH was performed on frozen tumors as previously described [17] using 44K microarrays (Agilent Technologies). Array CGH data have been deposited in Gene Expression Omnibus (GEO, $\underline{\text { http:// }}$ www.ncbi.nlm.nih.gov/geo/) and they are accessible through GEO Series accession number GSE14109.

\section{microRNA expression profiling}

We performed microRNA expression profiling starting from $100 \mathrm{ng}$ of total RNA and following the microRNA Microarray System protocol v.1.5 (Agilent Technologies). In dephosphorylation and ligation steps, 17 Units of Calf Intestine Alkaline Phosphatase (Takara Bio Inc., Shiga, Japan) and 20 Units of T4 RNA ligase (Takara Bio Inc.) have been used, respectively. Each sample was hybridized on Human microRNA Microarray v.2 (Agilent Technologies) containing probes for 723 human microRNAs. Slides were scanned by Agilent G2565BA scanner and images were processed by Feature Extraction software v.9.5.3.1 (Agilent Technologies). Tab-delimited text file were analyzed in R v.2.7.2 software environment http://www.rproject.org using the limma package v.2.14.16 of Bioconductor http://www.bioconductor.org. Only spots with signal minus background flagged as positive and significant were used in the following analysis as 'detected' spot. Probes with less than $50 \%$ of detected spots across all arrays, and arrays with a number of detected spots smaller than $50 \%$ of all spots on the array were removed. Background corrected intensities of replicated spots on each array were averaged. Data were then log2-transformed and normalized for between-array comparison using quantile normalization [18]. Differential expression analysis between "long-survivors" and "short-survivors" category was carried out using Student's $t$-test (unpaired, twotailed, unequal variance). MicroRNAs with P-values < 0.05 were selected for further analysis. Given the explorative nature of this study, no correction for multiple testing was applied in the screening procedure aimed at selecting multiple sets of microRNAs for subsequent hierarchical clustering analyses. The agglomerative hierarchical clusters, used to detect similarity relationships in microRNA log2-transformed expressions, were computed by the Euclidean distance between single vectors and the Ward method [19]. Microarray data have been deposited in GEO (Series accession number GSE16444).

\section{Functionally prediction of T-UCR signature}

T-UCR::microRNA sequence comparison was performed by using the Basic Local Alignment Search Tool available in the UCbase \& miRfunc database http://micro rna.osu.edu/UCbase4/[20]. Putative targets of microRNAs were determined by the miRBase Targets v.5 http://micro rna.sanger.ac.uk/targets/v5/ prediction algorithm.

\section{Results and Discussion \\ T-UCR expression profiling of NB tumors}

We investigated the expression of 481 T-UCRs by RTqPCR in two independent study groups of high-risk NB patients. Overall, 34 NBs were investigated, including 17 tumors of patients who died of disease and 17 samples of long-survivors. About $96 \%$ of T-UCRs were expressed in NB samples at variable levels, with the exception of 15 
transcripts (uc.82, uc.105, uc.160, uc.204, uc.213, uc.284, uc. 299, uc.314, uc.327, uc.344, uc.347, uc.357, uc.363, uc.398, uc.407) that were undetectable in all samples. Six T-UCRs (uc.88, uc.95, uc.157, uc.162, uc.361, uc.394) showed a $\mathrm{Cq}$ mean value above 35 and were therefore considered as not expressed. The majority of T-UCRs found in NB samples are nonexonic (53\%), whereas exonic and possibly exonic T-UCRs are transcribed at a lower frequency (23.3\% and $23.6 \%$, respectively).

\section{T-UCR signature of NBs}

T-UCR transcription levels were initially quantified in a set of 20 metastatic NBs of 8 short- and 12 long-survivors. Fifty-four of the 460 detectable T-UCRs (11.7\%) showed a differential expression between the two groups $(0.0004$ $<P<0.0491$ ) (see additional file 4: Table S2; additional file 5: Figure S1). The predictive value of the 54 differentially expressed on survival was analyzed in univariate model utilizing the thresholds defined by the ROC curves (see additional file 4: Table S2; additional file 6: Figure S2). The Kaplan-Meier curves showed that T-UCR expression levels above the cut-off value were significantly associated with a more favorable outcome in all 54 T-UCR for OS (0.0001 $<P<0.0369)$, and only in 48 T-UCRs for EFS $(0.0001<P<0.0491)$ (see additional file 4: Table S2; additional file 7: Figure S3).

Then, the T-UCR threshold risk-prediction model was tested in an independent cohort of 14 advanced stage NBs of 9 short- and 5 long-survivors. Kaplan-Meier survival analysis confirmed the significant association between higher expression level and better outcome for 28 T-UCRs (OS: $0.0007<\mathrm{P}<0.0399$, EFS: $0.0001<\mathrm{P}<0.0399$ ) (see additional file 4: Table S2; additional file 8: Figure S4).

Next we wondered whether accuracy in survival prediction could be improved by a combination of T-UCRs. In this respect, the significant $28 \mathrm{~T}$-UCRs were categorized as up- or down-expressed in each NB sample according to their respective cut-off values identified by ROC curves. ROC and Kaplan-Meier survival analyses (Figure 1) showed that at least 15 up-regulated T-UCRs are needed to discriminate $(P<0.0001)$ long- from short-survivors at the highest sensitivity (94.12\%) and specificity (94.12\%). MYCN gene status was tested by Cox regression analysis and it was removed during the backward selection, showing that the multi-T-UCR expression signature is a MYCNindependent predictor for OS and EFS in our cohort. These findings endorse the identification of a novel candidate prognostic molecular signature based on the expression profile of the T-UCRs in high-risk NB patients.

\section{T-UCR signature and genomic regions involved in NB}

On the basis of tumor genomic DNA availability, 29 (from 14 short- and 15 long-survivors) out of the 34 NBs were analyzed by array CGH (see additional file 1: Table S1; additional file 9: Figure S5). Tumors from the group of long-survivors showed 55 numerical (average 3.7 per subject) and 78 structural (average 5.2 per subject) chromosomal aberrations, mostly represented by $17 \mathrm{q}$ gain $(60 \%)$ and $11 \mathrm{q}$ loss (40\%). All tumors of short-survivors had at least one structural alteration (average 8.5 per subject). The most frequent changes were $17 \mathrm{q}$ gain $(100 \%), 1 \mathrm{p}$ loss (57\%), 2p gain $(57 \%), 11 q$ loss $(43 \%)$, 1q gain $(43 \%)$, and $3 p$ loss (29\%). Our results confirm that severe clinical courses are characterized by DNA changes at chromosomes $1,2,3,7,11$, and $17[5,6]$.

In order to investigate T-UCR alterations at genomic level, we associated the location of the 28 T-UCRs with the DNA imbalances identified in our cohort of samples. Fisher's test disclosed a significant $P$-value for UCRs mapping at 3q13.31 $(P=0.04)$, demonstrating that such UCRs (namely, uc.121 and uc.122) are more specifically associated with the corresponding genome location than expected by chance alone. According to this observation, one unfavorable NB (\#1506) shows 3q11.2-q29 loss, whereas two tumors of long-survivors (\#1889 and \#2056) harbor 3q12.2-qter and 3q13.2-q23 gain, respectively. In this respect, we did not identify any significant T-UCRenrichment within the more frequently observed regions of imbalance (i.e. 1p, 11q, 3p, 17q). In conclusion we hypothesize that the deregulation of T-UCR expression is not consistently associated with alterations at DNA level in the pathogenesis and progression of high-risk NB.

\section{Prediction of functional characterization of NB-associated T-UCRs}

The presence of a positive correlation between expression levels of exonic UCRs and host genes would suggest changes in primary transcription rates. It is noteworthy that five UCRs (uc.322, uc.323, uc.421, uc.423, and uc.452) up-regulated in long-survivors are located within three genes (SOX6, ZNF521, TSHZ3) that we found significantly (at a false discovery rate of 0.01 ) over-expressed in long-surviving subjects of an unrelated cohort of 76 highrisk NBs (personal microarray data, manuscript in preparation). According to these results, we speculate a positive enhancer activity on mRNA transcription for some TUCRs in NB, in agreement with previous observations $[21,22]$.

In order to investigate the post-transcriptional regulation of T-UCRs, we performed a genome-wide microRNA expression analysis in 32 out of 34 samples investigated for T-UCR levels. The expression dataset, after filtering, condensing by replicates average and control spots removal, contained 319 microRNAs and 31 eligible samples. Thirteen of the 319 (4.1\%) microRNAs showed differential expression between the two categories of tumors 

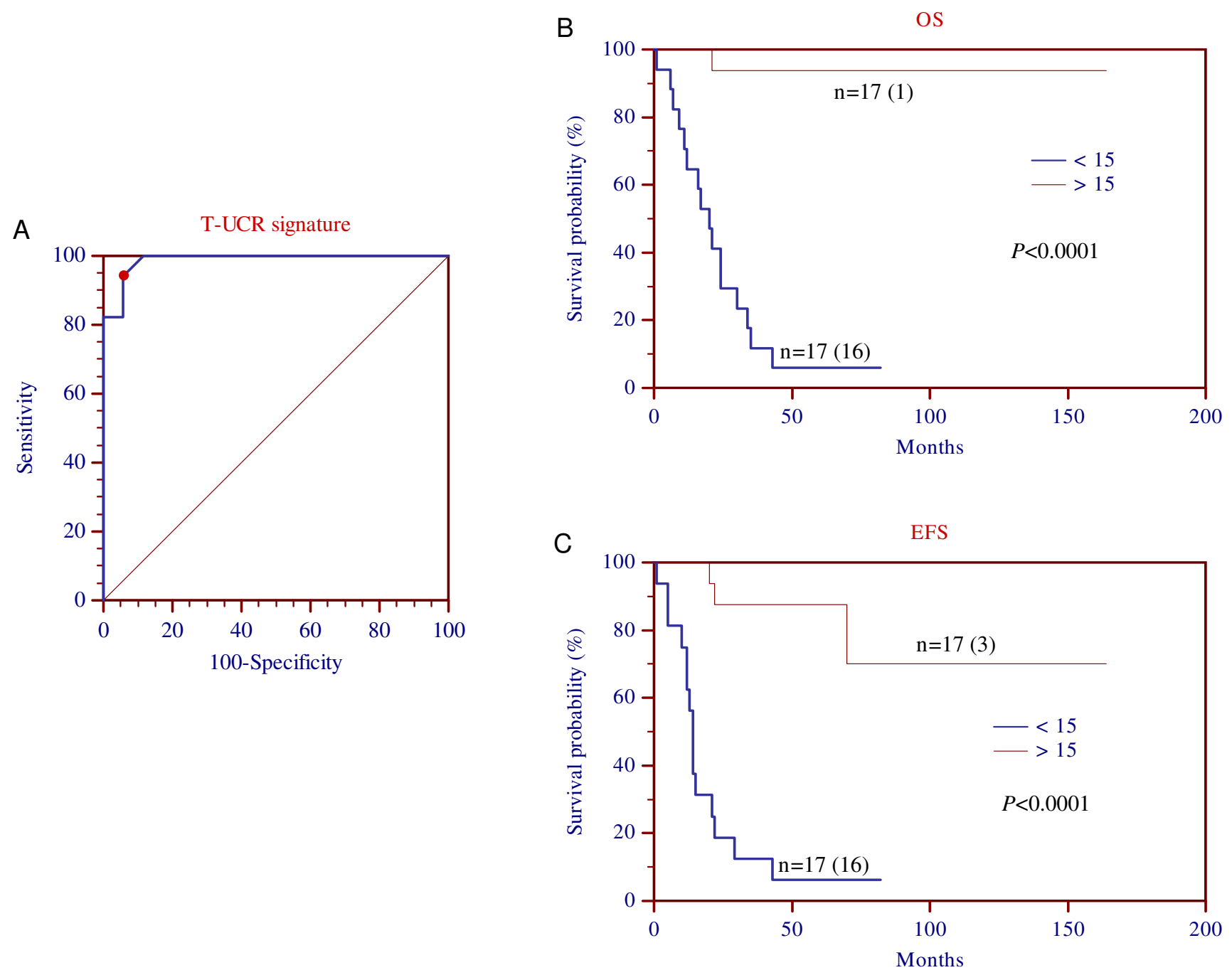

\section{Figure I}

(A) ROC curve. Sensitivity and specificity in predicting survival based on different numbers of T-UCRs whose expression levels are above the thresholds defined by each respective ROC curve. The point marked with the red dot represents the highest average of sensitivity and specificity at the cut-off value of I5 (AUC $=0.988, \mathrm{Z}$ statistic $=25.078, P=0.000 \mathrm{I})$. Kaplan-Meier analysis for (B) overall and (C) event-free survival of all 34 NBs. Comparison of survival probabilities of patients with $\geq 15$ or $<15$ up-regulated T-UCRs. In each graph are reported the number of patients in predicted subgroups and the number of patients with event (between brackets).

(Figure 2; additional file 10: Figure S6). Interestingly, $A L K$ tyrosine kinase receptor precursor mRNA is a putative target of hsa-miR-376c that is up-regulated in tumors of long-survivors. Although the targeting of the ALK 3'untranslated region by this microRNA remains to be experimentally validated, if true, this would suggest the importance for hsa-miR-376c to be induced in order to negate the effects of $A L K$ amplification in advanced NBs [23].

Intriguingly, 4 microRNAs down-regulated in tumors of long-survivors (hsa-miR-383, hsa-miR-548d-5p, hsa-miR-
939, hsa-miR-877*) target 3 genes implicated in neuronal differentiation (CHD5, FYN and NTRK1), that were recently reported to be over-expressed in low-risk tumors [7].

Since Calin et al. [10] demonstrated a negative regulation of T-UCRs by direct interaction with microRNAs, we argue that down-expression of the T-UCR signature in short-survivors may be due to the up-regulation of their own putative complementary microRNAs. Intriguingly, microRNA expression data confirmed our hypothesis: 5 out of the 13 microRNAs from the signature display their expression 


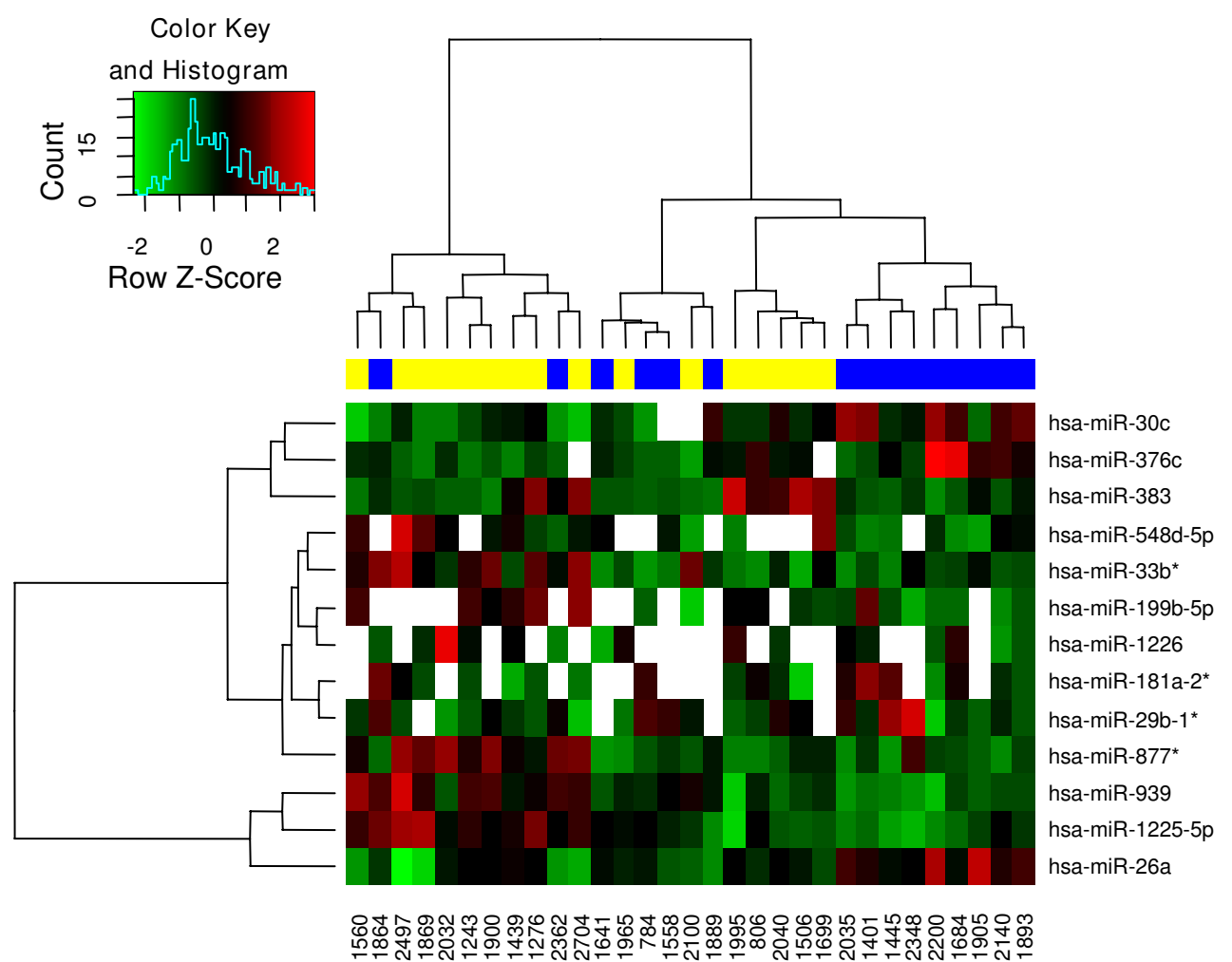

Figure 2

Hierarchically clustered heat map of differentially expressed microRNAs $(n=13)$ between the two tumors conditions. Non-detected signals are indicated in white. On top bar, short-survivors tumors (yellow) and long-survivor tumors (blue) are shown.

level that are negatively correlated with expression of 9 complementary UCRs able to differentiate between the two NB prognosis groups (Table 1). Sequence homologies give rise to 10 possible pairs, in agreement with previous reports on complex redundancy in regulatory mechanisms between microRNAs and T-UCRs [10].

Moreover, we speculate that a further plausible explanation for the directionality of expression of T-UCRs in longversus short-term survivors may be due to the contribution of an epigenetic regulation of T-UCR expression. This is supported by the fact that a CpG island methylator phenotype is present specifically in neuroblastoma with poor prognosis $[24,25]$, and that epigenetic mechanisms not only regulate coding genes but also non-coding RNAs, such as miRNAs [26]. According to these information, we retrieved the promoter sequences of T-UCR-host genes from DataBase of Transcriptional Start Sites (DBTSS, release 7.0, http://dbtss.hgc.jp/), where each promoter sequence contains $1 \mathrm{~kb}$ upstream sequence and $200 \mathrm{bp}$ sequence downstream from each transcription start site described. Then, we used the criteria and algorithm searcher at http://www.uscnorris.com/cpgislands2/ cpg.aspx [27] to obtain the CpG-island information. We found that 29 out the 37 T-UCRs of the signature (78\%) mapping within coding genes are associated with CpG islands in the promoter region of their own host genes (see additional file 11: Table S3). Therefore, like CpG island hypermethylation-mediated silencing of miRNAs with tumor suppressor features contribute to human cancer [28], we can argue that a global DNA hypermethylation events in unfavorable neuroblastomas may affect also T-UCR-host genes, and thus silence T-UCRs with a potential oncogenic role in metastatic NB tumors. Experimental validations will be performed in order to investigate the role of methylation in the regulation of T-UCR expression.

\section{Conclusions}

Our findings strongly suggest that a deregulation of the microRNA/T-UCR network may play an important role in NB. Functional studies are mandatory to clarify coding and noncoding RNA interactions in the pathogenesis of this tumor, and could therefore identify potential therapeutic targets.

The potential to use this information as the first T-UCR prognostic signature for high-risk neuroblastoma patients 
Table I: T-UCRs whose expression inversely correlates with complementary microRNAs differentially expressed in NB patients.

\begin{tabular}{|c|c|c|c|}
\hline $\begin{array}{l}\text { T- } \\
\text { UCR }\end{array}$ & $\begin{array}{l}\text { T-UCR expression in long- versus short- } \\
\text { survivors }\end{array}$ & $\begin{array}{c}\text { Complementary mature } \\
\text { microRNA }\end{array}$ & $\begin{array}{c}\text { microRNA expression in long- versus } \\
\text { short-survivors }\end{array}$ \\
\hline uc.209 & up-regulated & hsa-miR-877* & down-regulated \\
\hline uc.27I & up-regulated & hsa-miR-383 & down-regulated \\
\hline uc. 312 & up-regulated & $\begin{array}{c}\text { hsa-miR-877* } \\
\text { hsa-miR-548d-5p }\end{array}$ & down-regulated \\
\hline uc. 330 & up-regulated & hsa-miR-548d-5p & down-regulated \\
\hline uc.37I & up-regulated & hsa-miR-877* & down-regulated \\
\hline uc.4II & up-regulated & hsa-miR-33b* & down-regulated \\
\hline uc. 421 & up-regulated & hsa-miR-877* & down-regulated \\
\hline uc. 435 & up-regulated & hsa-miR-939 & down-regulated \\
\hline uc. 452 & up-regulated & hsa-miR-383 & down-regulated \\
\hline
\end{tabular}

must be tested in a larger, independent set of samples. Since immediate advantages of the qPCR strategy for TUCR signature evaluation are speed, cost-effectiveness, minimal required amounts of tumor RNA, and applicability as routine test, this pilot study should put the basis for future analyses in order to investigate i.e., whether the TUCR signature described here is a sensitive and specific genomic feature also in low- and intermediate-risk tumors.

\section{Abbreviations}

(T-UCRs): Transcribed-Ultra Conserved Regions; PCR (RT-qPCR): reverse transcription-quantitative real-time; (NB): neuroblastoma; (Cq): quantification cycle; (MIQE): Minimum Information for Publication of Quantitative Real-Time PCR Experiments; (ROC): Receiver Operating Characteristic; (OS): Overall survival; (EFS): Event free survival; (CGH): Comparative Genomic Hybridization; (GEO): Gene Expression Omnibus; (DBTSS): DataBase of Transcriptional Start Sites.

\section{Competing interests}

The authors declare that they have no competing interests.

\section{Authors' contributions}

PS developed the study design; supervised the preparation and quality assessment of RNAs, and the microarray experiments; participated in performing RT-qPCR reactions and data analysis; drafted the manuscript. SS carried out the preparation of RNAs, the quantification and assessment of transcript integrity, and the microarray experiments; participated in performing the RT-qPCR reactions. SC, FV, CDV performed the T-UCR qPCR reactions. SM performed microarray analysis. AG provided patients' clinical data. SB participated in data analysis and critical revision of the manuscript. GPT participated in study conception and critical revision of the manuscript. All authors read and approved the final manuscript.

\section{Additional material}

\section{Additional file 1}

Table S1. Clinical and biological characteristic of 34 NBs enrolled in the study.

Click here for file

[http://www.biomedcentral.com/content/supplementary/14712407-9-441-S1.PDF]

\section{Additional file 2}

Raw Cq data. Raw Cq values for each qPCR reaction, according to the Real-time PCR Data Markup Language (RDML).

Click here for file

[http://www.biomedcentral.com/content/supplementary/14712407-9-441-S2.XSL]

\section{Additional file 3}

$M I Q E$ checklist. Checklist according to the Minimum Information for Publication of Quantitative Real-Time PCR Experiments.

Click here for file

[http://www.biomedcentral.com/content/supplementary/14712407-9-441-S3.PDF]

\section{Additional file 4}

Table S2. T-UCRs differentially expressed between short- and long-survivors $(P<0.05)$. The table shows also results of ROC and survival analysis based on expression values of the 54 T-UCRs.

Click here for file

[http://www.biomedcentral.com/content/supplementary/14712407-9-441-S4.PDF]

\section{Additional file 5}

Figure S1. Box-and-Whisker plots of the comparison among values of TUCR expression by qPCR (normalized to $18 S$ rRNA, and after logarithmic transformation of original measures) between long-and short-survivors. Each box represents the values from the $25^{\text {th }}$ to $75^{\text {th }}$ percentile, the middle line represents the median, and a line extends from the minimum to the maximum value, excluding outliers which are displayed as blue dots. Nearby each outlier value is indicated the ID of the corresponding $N B$ sample. Relative expression data were multiplied by $10^{4}$.

Click here for file

[http://www.biomedcentral.com/content/supplementary/14712407-9-441-S5.PDF] 


\section{Additional file 6}

Figure S2. ROC curves based on qPCR results of the 54 T-UCRs differently expressed between long- and short-survivors. In each graph the point indicated with a red dot is the value corresponding to the highest average of sensitivity and specificity.

Click here for file

[http://www.biomedcentral.com/content/supplementary/14712407-9-441-S6.PDF]

\section{Additional file 7}

Figure S3. Kaplan-Meier overall and event-free survival curves of the first set of $20 \mathrm{NB}$ patients categorized according to T-UCR expression values (above or below the threshold defined by the respective ROC curve). In each graph are reported the number of patients in predicted subgroups and the number of patients with event (between brackets).

Click here for file

[http://www.biomedcentral.com/content/supplementary/1471-

2407-9-441-S7.PDF]

\section{Additional file 8}

Figure S4. Kaplan-Meier overall and event-free survival curves of the second set of 14 NB patients categorized according to T-UCR expression values (above or below the threshold defined in the first set of $20 \mathrm{NB}$ samples by the respective ROC curve). In each graph are reported the number of patients in predicted subgroups and the number of patients with event (between brackets).

Click here for file

[http://www.biomedcentral.com/content/supplementary/1471-

2407-9-441-S8.PDF]

\section{Additional file 9}

Figure S5. Structural chromosomal aberrations. The Figure shows percentages of gains (A) and losses (B) for each of the autosomal chromosomes detected by array CGH in tumors of 14 short- and 15 longsurvivors.

Click here for file

[http://www.biomedcentral.com/content/supplementary/1471-

2407-9-441-S9.PDF]

\section{Additional file 10}

Figure S6. Box plots of log2-transformed expression measures of the 13 microRNA differently expressed between long- and short-survivors. Each box represents the distribution of expression measured for "long-survivors" (blue, labeled by 0) and "short-survivors" (red, labeled by 1) tumor-category.

Click here for file

[http://www.biomedcentral.com/content/supplementary/14712407-9-441-S10.PDF]

\section{Additional file 11}

Table S3. T-UCRs associated with CpG islands in the promoter region of their own host genes.

Click here for file

[http://www.biomedcentral.com/content/supplementary/1471-

2407-9-441-S11.PDF]

\section{Acknowledgements}

This study was supported by Fondazione Italiana per la Lotta al Neuroblastoma, Ministero dell'Università, Ricerca Scientifica e Tecnologica, and Ministero della Salute/Regione Liguria (project: "Identification of tumor biomarkers through a biology-driven integrated approach"). We are grateful to surgeons, clinicians, pathologists of the Associazione Italiana Ematologia Oncologia Pediatrica (AIEOP) for providing tumor samples, to Silvia De Luca (Fondazione Italiana per la Lotta al Neuroblastoma, Genoa, Italy) for language revision, and to Barbara Banelli (Laboratory of Tumor Genetics, IST, Genoa, Italy) for helpful discussion. A special thank to Giorgio Canessa, Caterina Cremonesi and Carlo Costa (Eppendorf Italia, Milan, Italy) for technical assistance.

\section{References}

I. Maris JM, Hogarty MD, Bagatell R, Cohn SL: Neuroblastoma. Lancet 2007, 369:2106-2120.

2. London WB, Castleberry RP, Matthay KK, Look AT, Seeger RC, Shimada H, Thorner P, Brodeur G, Maris JM, Reynolds CP, Cohn SL: Evidence for an age cutoff greater than 365 days for neuroblastoma risk group stratification in the Children's Oncology Group. I Clin Oncol 2005, 23:6459-6465.

3. Tonini GP, Pistoia V: Molecularly guided therapy of neuroblastoma: a review of different approaches. Curr Pharm Des 2006, I 2:2303-2317.

4. Mosse YP, Greshock J, Weber BL, Maris JM: Measurement and relevance of neuroblastoma DNA copy number changes in the post-genome era. Cancer Lett 2005, 228:83-90.

5. Scaruffi P, Valent A, Schramm A, Astrahantseff K, Eggert A, Tonini GP. Application of microarray-based technology to neuroblastoma. Cancer Lett 2005, 228: I3-20.

6. Warnat P, Oberthuer A, Fischer M, Westermann F, Eils R, Brors B: Cross-study analysis of gene expression data for intermediate neuroblastoma identifies two biological subtypes. $B M C$ Cancer 2007, 7:89.

7. Vermeulen J, De Preter K, Naranjo A, Vercruysse L, Van Roy N, Hellemans J, Swerts K, Bravo S, Scaruffi P, Tonini GP, De Bernardi B, Noguera R, Piqueras M, Cañete A, Castel V, Janoueix-Lerosey I, Delattre O, Schleiermacher G, Michon J, Combaret V, Fischer M, Oberthuer A, Ambros PF, Beiske K, Bénard J, Marques B, Rubie H, Kohler J, Pötschger U, Ladenstein R, Hogarty MD, McGrady P, London WB, Laureys G, Speleman F, Vandesompele J: Predicting outcomes for children with neuroblastoma using a multigeneexpression signature: a retrospective SIOPEN/COG/GPOH study. Lancet Oncol 2009, 10:663-671.

8. Bilke S, Chen QR, Wei JS, Khan J: Whole Chromosome alterations predict survival in high-risk neuroblastoma without MYCN amplification. Clin Cancer Res 2008, 14:5540-5547.

9. Chen Y, Stallings RL: Differential Patterns of MicroRNA Expression in Neuroblastoma Are Correlated with Prognosis, Differentiation, and Apoptosis. Cancer Res 2007, 67:976-983.

10. Calin GA, Liu CG, Ferracin M, Hyslop T, Spizzo R, Sevignani C, Fabbri M, Cimmino A, Lee EJ, Wojcik SE, Shimizu M, Tili E, Rossi S, Taccioli C, Pichiorri F, Liu X, Zupo S, Herlea V, Gramantieri L, Lanza G, Alder H, Rassenti L, Volinia S, Schmittgen TD, Kipps TJ, Negrini M, Croce CM: Ultraconserved regions encoding ncRNAs are altered in human leukemias and carcinomas. Cancer Cell 2007, 1 2:215-229.

II. Bejerano G, Pheasant M, Makunin I, Stephen S, Kent WJ, Mattick JS, Haussler D: Ultraconserved elements in the human genome. Science 2004, 304: I32I-I325.

12. Brodeur GM, Pritchard J, Berthold F, Carlsen NL, Castel V, Castelberry RP, De Bernardi B, Evans AE, Favrot M, Hedborg F: Revisions of the international criteria for neuroblastoma diagnosis, staging, and response to treatment. J Clin Oncol 1993, II:1466-1477.

13. Shimada H, Ambros IM, Dehner LP, Hata J, Joshi VV, Roald B: Terminology and morphologic criteria of neuroblastic tumors: recommendations by the International Neuroblastoma Pathology Committee. Cancer 1999, 86:349-363.

14. Lefever S, Hellemans J, Pattyn F, Przybylski DR, Taylor C, Geurts R, Untergasser A, Vandesompele J: RDML: structured language and reporting guidelines for real-time quantitative PCR data. Nucleic Acids Res 2009, 37:2065-2069.

15. Bustin SA, Benes V, Garson JA, Hellemans J, Huggett J, Kubista M, Mueller R, Nolan T, Pfaffl MW, Shipley GL, Vandesompele J, Wittwer CT: The MIQE Guidelines: Minimum Information for Publi- 
cation of Quantitative Real-Time PCR Experiments. Clin Chem 2009, 55:6I I-622.

16. Griner PF, Mayewski RJ, Mushlin AI, Greenland P: Selection and interpretation of diagnostic tests and procedures. Principles and applications. Ann Intern Med I 981, 94:557-592.

17. Scaruffi P, Coco S, Cifuentes F, Albino D, Nair M, Defferrari R, Mazzocco K, Tonini GP: Identification and characterization of DNA imbalances in neuroblastoma by high-resolution oligonucleotide array comparative genomic hybridization. Cancer Genet Cytogenet 2007, 177:20-29.

18. Bolstad BM, Irizarry RA, Astrand M, Speed TP: A comparison of normalization methods for high density oligonucleotide array data based on bias and variance. Bioinformatics 2003, 19:185-193.

19. Ward $\mathrm{H}$ : Hierachical grouping to optimize an objective function. Journal of The American Statistical Association 1963, 58:236-244.

20. Taccioli C, Fabbri E, Visone R, Volinia S, Calin GA, Fong LY, Gambari R, Bottoni A, Acunzo M, Hagan J, lorio MV, Piovan C, Romano G, Croce CM: UCbase \& miRfunc: a database of ultraconserved sequences and microRNA function. Nucleic Acids Res 2009, 37:D4I-D48.

21. Pennacchio LA, Ahituv N, Moses AM, Prabhakar S, Nobrega MA, Shoukry M, Minovitsky S, Dubchak I, Holt A, Lewis KD, Plajzer-Frick I, Akiyama J, De Val S, Afzal V, Black BL, Couronne O, Eisen MB, Visel $A$, Rubin EM: In vivo enhancer analysis of human conserved non-coding sequences. Nature 2006, 444:499-502.

22. Visel A, Prabhakar S, Akiyama JA, Shoukry M, Lewis KD, Holt A, Plajzer-Frick I, Afzal V, Rubin EM, Pennacchio LA: Ultraconservation identifies a small subset of extremely constrained developmental enhancers. Nat Genet 2008, 40:158-160.

23. Carén $\mathrm{H}$, Abel $F$, Kogner $\mathrm{P}$, Martinsson $\mathrm{T}$ : High incidence of DNA mutations and gene amplifications of the ALK gene in advanced sporadic neuroblastoma tumours. Biochem J 2008 , 416:153-159.

24. Abe M, Ohira M, Kaneda A, Yagi Y, Yamamoto S, Kitano Y, Takato T, Nakagawara A, Ushijima T: CpG island methylator phenotype is a strong determinant of poor prognosis in neuroblastomas. Cancer Res 2005, 65:828-834.

25. Banelli B, Bonassi S, Casciano I, Mazzocco K, Di Vinci A, Scaruffi P, Brigati C, Alemanni G, Borzì L, Tonini GP, Romani M: Outcome prediction and risk assessment by quantitative pyrosequencing methylation analysis of the SFN gene in advanced stage, high-risk, neuroblastic tumor patients. Int J Cancer 20I0, 126:656-68

26. Lujambio A, Calin GA, Villanueva A, Ropero S, Sanchez-Cespedes M, Blanco D, Montuenga LM, Rossi S, Nicoloso MS, Faller WJ, Gallagher WM, Eccles SA, Croce CM, Esteller M: A microRNA DNA methylation signature for human cancer metastasis. Proc Natl Acad Sci USA 2009, 105: |3556-1356।.

27. Takai $D$, Jones PA: Comprehensive analysis of CpG islands in human chromosomes 21 and 22. Proc Natl Acad Sci USA 2002, 99:3740-3745.

28. Lujambio A, Esteller M: How epigenetics can explain human metastasis. Cell Cycle 2009, 8:377-382.

\section{Pre-publication history}

The pre-publication history for this paper can be accessed here:

http://www.biomedcentral.com/1471-2407/9/441/pre pub
Publish with Biomed Central and every scientist can read your work free of charge

"BioMed Central will be the most significant development for disseminating the results of biomedical research in our lifetime. "

Sir Paul Nurse, Cancer Research UK

Your research papers will be:

- available free of charge to the entire biomedical community

- peer reviewed and published immediately upon acceptance

- cited in PubMed and archived on PubMed Central

- yours - you keep the copyright
BioMedcentral 